\title{
Unitarity of the box diagram
}

\section{Roji Pius ${ }^{a}$ and Ashoke Sen ${ }^{b}$}

${ }^{a}$ Perimeter Institute for Theoretical Physics, Waterloo, ON N2L 2Y5, Canada

${ }^{b}$ Harish-Chandra Research Institute, HBNI, Chhatnag Road, Jhusi, Allahabad 211019, India

E-mail: rpius@perimeterinstitute.ca, sen@hri.res.in

ABSTRACT: The complete proof of cutting rules needed for proving perturbative unitarity of quantum field theories usually employs the largest time equation or old fashioned perturbation theory. None of these can be generalized to string field theory that has non-local vertices. In arXiv: 1604.01783 we gave a proof of cutting rules in string field theory, which also provides an alternative proof of cutting rules in ordinary quantum field theories. In this note we illustrate how this works for the box diagram of $\phi^{4}$ field theory, avoiding the contributions from anomalous thresholds.

KEYwords: Scattering Amplitudes, String Field Theory

ArXiv EPRINT: 1805.00984 


\section{Contents}

1 Introduction and summary 1

2 The issues 2

3 Unitarity of the box diagram 3

4 Unitarity of tree diagrams $\quad 14$

\section{Introduction and summary}

Cutkosky's diagrammatic analysis shows that the discontinuities of a Feynman diagram across the 'normal threshold' singularities produce the result needed for unitarity of the S-matrix [1, 2]. However typically a Feynman diagram possesses many other Landau singularities e.g. anomalous thresholds at one loop [3, 4] and more complicated singularities at higher loop, and there are discontinuities associated with these singularities as well. A recent discussion on these may be found in $[5,6]$. For this reason the standard approach to proving the cutting rules needed for unitarity makes use of indirect methods e.g. the largest time equation $[7,8]$ or old fashioned perturbation theory [9] based on time ordered diagrams. A recent analysis along similar line, suitable for dealing with vertices with finite number of time derivatives, can be found in [10].

Unfortunately these approaches are not suitable for proving the cutting rules for the Feynman diagrams arising in string field theory, since the vertices are non-local, not only in space but also in time, involving exponentials of quadratic functions of momenta. For this reason in [11] we developed a different approach to proving the cutting rules in such theories based on direct analysis of Feynman diagrams. ${ }^{1}$ Although originally developed for string field theory, this approach also gives an alternative, diagrammatic proof of cutting rules in ordinary quantum field theories. Another approach to proving unitarity in local theories by directly dealing with momentum space Feynman diagrams was suggested in [13]. We suspect that this approach is closely related to the one described in [11], but the precise relation is not clear at present.

Since [11] gave an iterative proof of the cutting rules to all orders in perturbation theory, the analysis was necessarily somewhat abstract. In this paper we complement the analysis of [11] by showing how the method works for establishing the cutting rules for the box diagram that appears in the computation of one loop eight point amplitude in $\phi^{4}$ field theory. We emphasize however that the purpose of this note is not to prove unitarity of the

\footnotetext{
${ }^{1}$ There have been two recent papers $[6,12]$ on unitarity of non-local field theories of the kind that arise in string field theory, but both seem to only focus on the analysis of discontinuity across normal thresholds.
} 
box diagram for which there are many derivations. The goal is to illustrate how the iterative all order diagrammatic proof of the cutting rules given in [11] works for the box diagram.

The rest of the paper is organized as follows. In section 2 we discuss some general issues that arise while trying to prove unitarity of amplitudes written as momentum space integrals. In section 3, which is the main body of the paper, we show how the method developed in [11] is used to prove cutting rules for the box diagram of $\phi^{4}$ field theory. This proof assumes the validity of cutting rules for connected and disconnected tree diagrams. For completeness, in section 4 we give a proof of cutting rules for tree diagrams, again by making use of the general method described in [11]. We shall use mostly plus signature for the space-time metric and denote a $D$ dimensional momentum as $\left(\ell^{0}, \vec{\ell}\right)$ where $\ell^{0}$ denote the time component and $\vec{\ell}$ denotes the spatial components.

\section{The issues}

In this section we shall briefly discuss the issues that plague the proof of unitarity directly in momentum space. The singularities of a Feynman diagram are associated with Landau singularities where the integrand has poles due to certain number of internal propagators going on-shell and furthermore the integration contour over loop momenta are pinched, i.e. it is not possible to move away from the poles by deforming the integration contour in the complex loop momentum plane. At such singularities, the integral typically has a branch cut, leading to a discontinuity of the amplitude across the branch cut. In [1] Cutkosky gave a general formula for computing the discontinuity across a given threshold singularity. If a certain number of propagators go on-shell at a singularity, then the discontinuity in the amplitude from the corresponding branch cut is computed by replacing the $i /\left(-k^{2}-m^{2}+i \epsilon\right)$ factor in each of these propagators by $2 \pi \delta\left(-k^{2}-m^{2}\right)$.

Let us consider a singularity where the on-shell internal propagators are such that together they can be interpreted as an intermediate state in the original amplitude, e.g. if the set consists of the propagators carrying momenta $\ell$ and $p_{A}+p_{B}-\ell$ in figure 1 . (We are assuming that the incoming particles come from the left and the outgoing particles move to the right.) Branch points associated with such singularities are known as normal thresholds. In this case the discontinuity computed from Cutkosky's formula can be regarded as a product of two on-shell amplitudes, integrated over the phase space of the intermediate states. Therefore this looks similar to a contribution to $-i T^{\dagger} T=-i T^{\dagger}|n\rangle\langle n| T$ that is needed for unitarity of the T-matrix - related to the S-matrix via $S=1-i T$. Such contributions are usually represented as cut diagrams, where the cut is a single line that divides the diagram into a left half and a right half, with the cut propagators representing the on-shell propagators.

While this goes a long way towards proving unitarity of the theory, there are some missing ingradients:

1. A given Feynman diagram may have singularities other than normal threshold, e.g. if the propagators carrying momenta $\ell, \ell+p_{C}$ and $p_{A}-\ell$ in figure 1 were on-shell at the singularity. Such singularities are known as anomalous thresholds. Cutkosky's 
formula can still be used to compute the discontinuity across such branch points; however in this case the on-shell states cannot collectively be regarded as an intermediate state in the sum $-i T^{\dagger}|n\rangle\langle n| T$.

2. In computing $-i T^{\dagger} T$ we need to reverse the signs of $i \epsilon$ in the propagators of the amplitude to the right of the cut so that it represents a matrix element of $T^{\dagger}$. This does not follow from Cutkosky's formula for discontinuity.

While for any specific graph one can do a more detailed analysis taking into account all these effects, the general proof of unitarity based on this approach becomes cumbersome. These problems were overcome in $[7,8]$ where a different proof of unitarity was given based on the 'largest time equation'. An alternative proof was given in [9] based on the old fashioned perturbation theory. However unlike Cutkosky's original analysis, which did not depend on the detailed structure of the vertices as long as they do not introduce additional singularities at finite momentum, the analysis of [7-9] requires working in coordinate space where at least for the time coordinate the propagator and vertices are expressed in the position space instead of the momentum space. Unfortunately for string field theory, for which the vertices are exponentials of quadratic functions of momenta, there is no convenient representation of the vertices in the coordinate space. Therefore the analysis of $[7-9]$ do not apply.

This difficulty was overcome in [11] that expressed $T-T^{\dagger}$ as a sum over cuts diagrams by working directly in momentum space. Furthermore the part of the contribution to the right of the cut was shown to be hermitian conjugated, representing a contribution to $T^{\dagger}$. This method is well-suited for string field theory and other non-local theories, but also for ordinary quantum field theories with local vertices, giving an alternative proof of unitarity. In the next two sections we shall illustrate how this works for the box diagram and tree diagrams in $\phi^{4}$ theory.

\section{Unitarity of the box diagram}

Usually in $\phi^{4}$ theory with interaction term $-(\lambda / 4 !) \int d^{D} x \phi^{4}$ one takes the propagator of momentum $k$ to be $-i /\left(k^{2}+m^{2}-i \epsilon\right)$ and the vertex to be $-i \lambda / 4$ !. Furthermore in the computation of the T-matrix we have an overall factor of $i$. However, as in [11], we shall use a slightly different but equivalent convention where for computation of the T-matrix we use the following rules:

1. The propagator of momentum $k$ is given by $1 /\left(-k^{2}-m^{2}+i \epsilon\right)$.

2. The vertex is given by $\lambda / 4$ !.

3. For each loop integral we have a factor of $i$.

4. If the diagram has $n_{c}$ disconnected components then we have a factor of $i^{1-n_{c}}$.

We shall drop the overall momentum conserving delta function $(2 \pi)^{D} \delta^{(D)}\left(\sum_{i} p_{i}\right)$, associated with each connected component, from the expressions for the amplitudes. The 


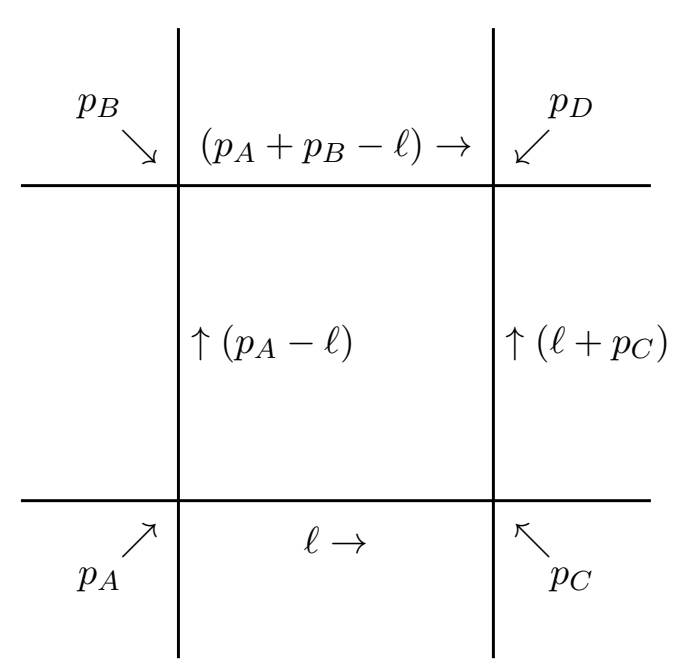

Figure 1. The box diagram of the eight point amplitude in $\phi^{4}$ theory. The external momenta $p_{A}, p_{B}, p_{C}, p_{D}$ entering at the four vertices are taken to be positive if ingoing.

space-time dimension $D=d+1$ will be chosen such that the box diagram of figure 1 has no ulltra-violet divergence. This requires $D \leq 7$.

With this convention the contribution of the box diagram shown in figure 1 to the T-matrix is given by,

$$
\begin{aligned}
I\left(p_{A}, p_{B}, p_{C}, p_{D}\right)= & \frac{i}{2} \lambda^{4} \int \frac{d^{D} \ell}{(2 \pi)^{D}}\left\{-\ell^{2}-m^{2}+i \epsilon\right\}^{-1}\left\{-\left(\ell+p_{C}\right)^{2}-m^{2}+i \epsilon\right\}^{-1} \\
& \left\{-\left(p_{A}-\ell\right)^{2}-m^{2}+i \epsilon\right\}^{-1}\left\{-\left(p_{A}+p_{B}-\ell\right)^{2}-m^{2}+i \epsilon\right\}^{-1}
\end{aligned}
$$

$p_{A}, p_{B}, p_{C}$ and $p_{D}$ denote net external momenta entering the vertices. In this convention an outgoing particle will have negative $p^{0}$. Since each of $p_{A}, p_{B}, p_{C}$ and $p_{D}$ receives contribution from two incoming or outgoing external states, they can be space-like or timelike and arbitrarily large in magnitude. Therefore all the singularities that can appear in the box diagram can be present here. In particular by taking $p_{A}$ to be a large time-like momentum we can mimick the case of a massive external particle above the threshold of production of a pair of $\phi$ particles - this is the situation in which the anomalous threshold is commonly discussed. ${ }^{2}$

Our goal will be to compute the quantity

$$
\mathcal{D} \equiv I\left(p_{A}, p_{B}, p_{C}, p_{D}\right)-I\left(-p_{A},-p_{B},-p_{C},-p_{D}\right)^{*}
$$

that represents a contribution to $T-T^{\dagger}$. We write down the expression for $I\left(-p_{A},-p_{B}\right.$, $\left.-p_{C},-p_{D}\right)^{*}$ by taking the complex conjugate of (3.1) and changing the signs of all the external momenta. Making a change of variables $\ell \rightarrow-\ell$ in the resulting expression,

\footnotetext{
${ }^{2}$ In fact we do not even need to assume in our analysis that the external momenta are on-shell.
} 
we have

$$
\begin{aligned}
I\left(-p_{A},-p_{B},-p_{C},-p_{D}\right)^{*}= & -\frac{i}{2} \lambda^{4} \int \frac{d^{D} \ell}{(2 \pi)^{D}}\left\{-\ell^{2}-m^{2}-i \epsilon\right\}^{-1}\left\{-\left(\ell+p_{C}\right)^{2}-m^{2}-i \epsilon\right\}^{-1} \\
& \left\{-\left(p_{A}-\ell\right)^{2}-m^{2}-i \epsilon\right\}^{-1}\left\{-\left(p_{A}+p_{B}-\ell\right)^{2}-m^{2}-i \epsilon\right\}^{-1} .
\end{aligned}
$$

This gives

$$
\begin{aligned}
\mathcal{D}= & \frac{i}{2} \lambda^{4} \int \frac{d^{d} \ell}{(2 \pi)^{d}} \int \frac{d \ell^{0}}{2 \pi}\left[\left\{-\ell^{2}-m^{2}+i \epsilon\right\}^{-1}\left\{-\left(\ell+p_{C}\right)^{2}-m^{2}+i \epsilon\right\}^{-1}\right. \\
& \left\{-\left(p_{A}-\ell\right)^{2}-m^{2}+i \epsilon\right\}^{-1}\left\{-\left(p_{A}+p_{B}-\ell\right)^{2}-m^{2}+i \epsilon\right\}^{-1} \\
& +\left\{-\ell^{2}-m^{2}-i \epsilon\right\}^{-1}\left\{-\left(\ell+p_{C}\right)^{2}-m^{2}-i \epsilon\right\}^{-1} \\
& \left.\left\{-\left(p_{A}-\ell\right)^{2}-m^{2}-i \epsilon\right\}^{-1}\left\{-\left(p_{A}+p_{B}-\ell\right)^{2}-m^{2}-i \epsilon\right\}^{-1}\right] .
\end{aligned}
$$

Unitarity of the S-matrix demands that $T-T^{\dagger}$ must be equal to $-i T^{\dagger} T$. This translates to the cutting rules which tell us that $\mathcal{D}$ is given by the sum over all cuts of the box diagram, with the following rules for evaluating a cut diagram:

1. A cut must divide the diagram into the left half and the right half, with the convention that the incoming particles come from the left and the outgoing particles travel to the right.

2. A cut propagator corresponds to the replacement:

$$
P(k) \equiv \frac{1}{-k^{2}-m^{2}+i \epsilon} \quad \Rightarrow \quad P_{c}(k) \equiv-2 \pi i \delta\left(-k^{2}-m^{2}\right) \theta\left(k^{0}\right),
$$

where $k$ denotes the momentum flowing along the propagator from the left side of the cut to the right side. The $-i$ factor in the expression for $P_{c}$ may seem unfamiliar, but in our convention this combines with the factor of $i$ from loop integral to give the correct integration measure over the phase space.

3. The part of the amplitude to the right of the cut is replaced by its hermitian conjugate - involving complex conjugation and reversal of the signs of all external momenta.

4. Cut on an external line has no effect.

5. If a cut diagram has $n_{L}$ disconnected components on the left of the cut and $n_{R}$ disconnected components on the right of the cut, then it should be multiplied by an additional factor of $(-1)^{n_{R}-1}$. This factor is needed to ensure that cutting rules lead to the unitarity relation $T-T^{\dagger}=-i T^{\dagger} T[11]$.

We shall first prove that for fixed $\vec{\ell}$ in (3.4), the contribution to $\mathcal{D}$ from the $\ell^{0}$ integral vanishes unless the integration contour is pinched between two singularities. ${ }^{3}$ For this we

\footnotetext{
${ }^{3}$ As in [11], we shall only allow deformations of loop energy integration contour into the complex plane, but keep the integration contours for spatial components of the loop momenta always along the real axes.
} 
deform the $\ell^{0}$ integral to $\infty$ in the lower half plane for the first term inside the square bracket in (3.4) and to $\infty$ in the upper half plane for the second term in the square bracket in (3.4), picking up residues from the poles that the contour passes through during the deformation. Since the poles of the first term are complex conjugates of the poles in the second term, we pick residues from exactly the same set of poles with $i \epsilon$ replaced by $-i \epsilon$. Furthermore in the first term the poles are traversed in the clockwise direction while in the second term the poles are traversed in the anti-clockwise direction. As long as there are no nearby poles, we can set $\epsilon=0$ while evaluating these residues. In this case their contributions exactly cancel. This argument breaks down if the contours are pinched since the residues diverge as $\epsilon \rightarrow 0$, and we have to carefully take the limit to see if there is any left-over contribution after we combine the results of the two terms. ${ }^{4}$

The pinch singularities occur when a pair of poles in the $\ell^{0}$ plane approach the integration contour from the opposite sides. Therefore in the $\vec{\ell}$ space they occur on a subspace of codimension 1 or higher (if more than two poles approach the same point). We shall call this the pinched subspace. We shall focus on the integration over a small neighborhood $\mathcal{R}$ of the pinched subspace. $\mathcal{R}$ is a $(D-1)$ dimensional space, i.e. of codimension zero. We denote by $\mathcal{R}^{\prime}$ the image of $\mathcal{R}$ under $\vec{\ell} \rightarrow-\vec{\ell}$, and consider the quantity

$$
\mathcal{D}_{\mathcal{R}}=A-A^{*},
$$

where

$$
\begin{aligned}
A= & \frac{i}{2} \lambda^{4} \int_{\mathcal{R}} \frac{d^{d} \ell}{(2 \pi)^{d}} \int \frac{d \ell^{0}}{2 \pi}\left\{-\ell^{2}-m^{2}+i \epsilon\right\}^{-1}\left\{-\left(\ell+p_{C}\right)^{2}-m^{2}+i \epsilon\right\}^{-1} \\
& \left\{-\left(p_{A}-\ell\right)^{2}-m^{2}+i \epsilon\right\}^{-1}\left\{-\left(p_{A}+p_{B}-\ell\right)^{2}-m^{2}+i \epsilon\right\}^{-1}
\end{aligned}
$$

and $A^{*}$ is obtained from $A$ by

1. replacing $\mathcal{R}$ by $\mathcal{R}^{\prime}$,

2. reversing the signs of all the external momenta, and

3. complex conjugation.

We shall prove that $\mathcal{D}_{\mathcal{R}}$ is given by the sum over cuts of the contributions to $A$. The full cutting rule is then obtained by adding the contributions from each small region $\mathcal{R}$ of this type.

In this section we shall prove the cutting rule for $\mathcal{D}_{\mathcal{R}}$ assuming that it holds for all tree diagrams - including disconnected ones. This analysis will follow closely the one given in section 5.2.3 of [11] for one vertex irreducible diagrams. In the next section we shall describe the proof of cutting rules for connected and disconnected tree diagrams.

Now for $\vec{\ell} \in \mathcal{R}$ a certain number of propagators become nearly on-shell when $\ell^{0}$ takes the value where its integration contour is nearly pinched. Since for small $\mathcal{R}, \ell^{0}$ lies within

\footnotetext{
${ }^{4}$ For string field theory the $\ell^{0}$ integration contour for both terms have their ends fixed at $\pm i \infty$ [11]. However a similar cancellation occurs for these contours as well. In this case bad behaviour in some directions at $\infty$ prevents us from deforming the contours to $\infty$, but the relevant contours can be deformed to each other.
} 


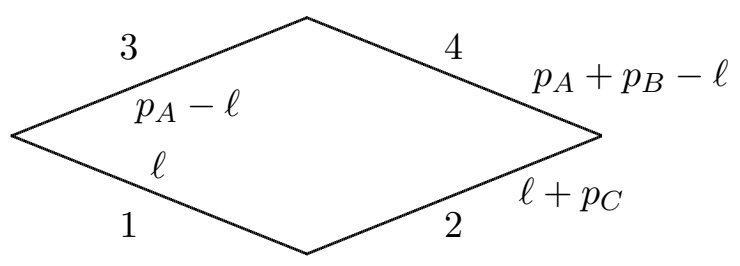

Figure 2. The reduced diagram of figure 1 associated with $\mathcal{R}$ when at the pinch all propagators are nearly on-shell and the energies carried by the propagators lie in the range (3.8). The internal lines are drawn in a way so that at the pinch energy flows from the left to the right along each of the propagators. Since four propagators become on-shell at the pinch, the pinched subspace has comension 4 , and $\mathcal{R}$ is a small neighborhood of this codimension 4 subspace.

a small range at the pinch, we can assign definite signs to the energies carried by each internal propagator at the pinch. We shall now associate with the region $\mathcal{R}$ a reduced diagram that is obtained from the original diagram by shrinking to points all propagators that are not nearly on-shell near the pinch. Furthermore we shall draw the nearly on-shell propagators such that energy flows from left to right near the pinch. For definiteness, and to consider a situation of maximal complexity, we shall consider a region $\mathcal{R}$ for which all four internal propagators are nearly on-shell at the pinch, and ${ }^{5}$

$$
\ell^{0}>0, \quad \ell^{0}+p_{C}^{0}>0, \quad p_{A}^{0}-\ell^{0}>0, \quad p_{A}^{0}+p_{B}^{0}-\ell^{0}>0,
$$

at the pinch. The corresponding reduced diagram is shown in figure 2. We have dropped the external legs from this diagram to avoid cluttering. We also number the propagators carrying momenta $\ell, \ell+p_{C}, p_{A}-\ell$ and $p_{A}+p_{B}-\ell$ by $1,2,3$ and 4 respectively. It is easy to see that the corresponding integral for $A^{*}$, after making a change of integration variable $\ell^{0} \rightarrow-\ell^{0}$, will be pinched at the same value of $\ell^{0}$.

Let us denote by $P(k)$ the propagator with momentum $k$ :

$$
P(k) \equiv \frac{1}{-k^{2}-m^{2}+i \epsilon}=\frac{1}{k^{0}-\sqrt{\vec{k}^{2}+m^{2}}+i \varepsilon} \frac{1}{k^{0}+\sqrt{\vec{k}^{2}+m^{2}}-i \varepsilon},
$$

where $\varepsilon$ is positive for positive $\epsilon$. Therefore we can express (3.7) as

$$
A=\frac{i}{2} \lambda^{4} \int_{\mathcal{R}} \frac{d^{d} \ell}{(2 \pi)^{d}} \int \frac{d \ell^{0}}{2 \pi} P(\ell) P\left(\ell+p_{C}\right) P\left(p_{A}-\ell\right) P\left(p_{A}+p_{B}-\ell\right) .
$$

Since the pinch is assumed to occur at the positive values of $\ell^{0}, \ell^{0}+p_{C}^{0}, p_{A}^{0}-\ell^{0}$ and $p_{A}^{0}+p_{B}^{0}-\ell^{0}$, the relevant poles of the propagators that take part in pinching the contour, are at

$$
\begin{array}{ll}
\ell^{0}=\sqrt{\vec{\ell}^{2}+m^{2}}-i \varepsilon, & \ell^{0}=-p_{C}^{0}+\sqrt{\left(\vec{\ell}+\vec{p}_{C}\right)^{2}+m^{2}}-i \varepsilon, \\
\ell^{0}=p_{A}^{0}-\sqrt{\left(\vec{p}_{A}-\vec{\ell}\right)^{2}+m^{2}}+i \varepsilon, & \ell^{0}=p_{A}^{0}+p_{B}^{0}-\sqrt{\left(\vec{p}_{A}+\vec{p}_{B}-\vec{\ell}\right)^{2}+m^{2}}+i \varepsilon .
\end{array}
$$

\footnotetext{
${ }^{5} \mathrm{~A}$ similar analysis can be carried out for all other reduced diagrams.
} 
Note that at the pinch the poles from the propagators 1 and 2 are in the lower half $\ell^{0}$ plane while the poles from the other propagators are in the upper half $\ell^{0}$ plane. Therefore while deforming the $\ell^{0}$ contour to the lower half plane, we shall pick up residues from the poles of the propagators 1 and 2 at the pinch. For this reason the set $\{1,2\}$ will play a special role in our analysis.

We now define

$$
P^{\prime}(k) \equiv \frac{1}{k^{0}-\sqrt{\vec{k}^{2}+m^{2}}-i \varepsilon} \frac{1}{k^{0}+\sqrt{\vec{k}^{2}+m^{2}}-i \varepsilon}=P(k)-P_{c}(k),
$$

where

$$
P_{c}(k) \equiv-2 \pi i \delta\left(-k^{2}-m^{2}\right) \theta\left(k^{0}\right),
$$

is the cut propagator. It follows from (3.12) that

$$
P(\ell) P\left(\ell+p_{C}\right)=P^{\prime}(\ell) P^{\prime}\left(\ell+p_{C}\right)+P_{c}(\ell) P\left(\ell+p_{C}\right)+P(\ell) P_{c}\left(\ell+p_{C}\right)-P_{c}(\ell) P_{c}\left(\ell+p_{C}\right) .
$$

Using this in (3.10) we can express $A$ as

$$
A=\widehat{A}+A^{(1)}+A^{(2)}-A^{(12)},
$$

where

$$
\begin{aligned}
\widehat{A} & =\frac{i}{2} \lambda^{4} \int_{\mathcal{R}} \frac{d^{d} \ell}{(2 \pi)^{d}} \int \frac{d \ell^{0}}{2 \pi} P^{\prime}(\ell) P^{\prime}\left(\ell+p_{C}\right) P\left(p_{A}-\ell\right) P\left(p_{A}+p_{B}-\ell\right), \\
A^{(1)} & =\frac{i}{2} \lambda^{4} \int_{\mathcal{R}} \frac{d^{d} \ell}{(2 \pi)^{d}} \int \frac{d \ell^{0}}{2 \pi} P_{c}(\ell) P\left(\ell+p_{C}\right) P\left(p_{A}-\ell\right) P\left(p_{A}+p_{B}-\ell\right), \\
A^{(2)} & =\frac{i}{2} \lambda^{4} \int_{\mathcal{R}} \frac{d^{d} \ell}{(2 \pi)^{d}} \int \frac{d \ell^{0}}{2 \pi} P(\ell) P_{c}\left(\ell+p_{C}\right) P\left(p_{A}-\ell\right) P\left(p_{A}+p_{B}-\ell\right), \\
A^{(12)} & =\frac{i}{2} \lambda^{4} \int_{\mathcal{R}} \frac{d^{d} \ell}{(2 \pi)^{d}} \int \frac{d \ell^{0}}{2 \pi} P_{c}(\ell) P_{c}\left(\ell+p_{C}\right) P\left(p_{A}-\ell\right) P\left(p_{A}+p_{B}-\ell\right) .
\end{aligned}
$$

In writing (3.17)-(3.19) we have used the notation of [11] in which $A^{\left(i_{1} \cdots i_{n}\right)}$ is obtained from $A$ by replacing the $i_{1}, \cdots i_{n}$-th propagators by cut propagators. The diagrammatic representations of $A^{(1)}, A^{(2)}$ and $A^{(12)}$ have been shown in figures 3-5 with the thick vertical line across a propagator representing a cut propagator $P_{c}$. We emphasize that these are not yet cut diagrams as the cut does not divide the graph into a left half and a right half, and we neither reverse the momenta not complex conjugate any part of the graph. Instead these should be regarded as tree amplitudes since a cut propagator can be regarded as a pair of incoming and outgoing lines with identical momentum. By a similar manipulation we can express $A^{*}$ as

$$
A^{*}=\widehat{A}^{*}+A^{(1) *}+A^{(2) *}-A^{(12) *} .
$$

We now note that in the expression (3.16) for $\widehat{A}$ the relevant poles in the $\ell^{0}$ plane, responsible for the pinch in the original amplitude $A$, are all in the upper half plane, since 


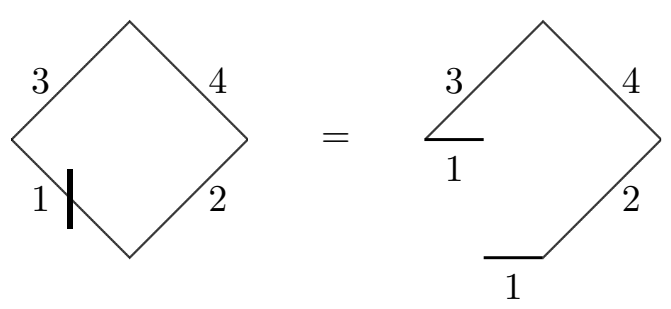

Figure 3. Representation of $A^{(1)}$. On the left hand side the vertical line through propagator 1 represents that it is a cut propagator. On the right hand side this is made explicit by replacing the cut propagator by a pair of external lines — one outgoing and one incoming.

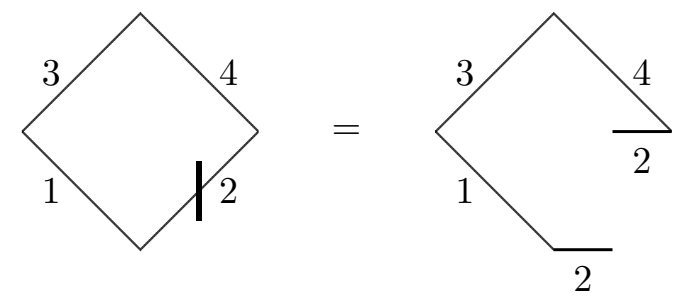

Figure 4. Representation of $A^{(2)}$.

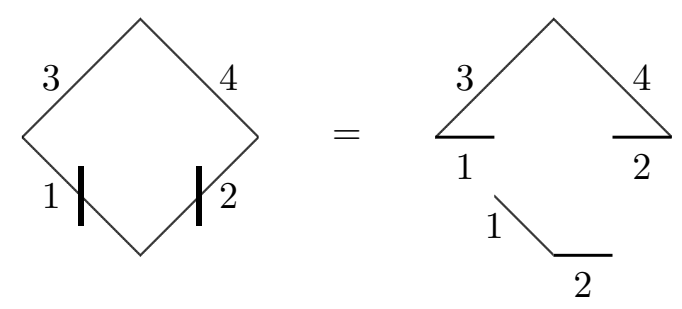

Figure 5. Representation of $A^{(12)}$.

in the locations of the poles of the integrand, the signs of the $i \varepsilon$ in the first line of (3.11) are reversed. Therefore the $\ell^{0}$ contour is not pinched, and by our previous argument,

$$
\widehat{A}-\widehat{A}^{*}=0 .
$$

On the other hand, since $A^{(1)}, A^{(2)}$ and $A^{(12)}$ are tree amplitudes, and since we are assuming that cutting rules hold for tree amplitudes, $A^{(1)}-A^{(1) *}, A^{(2)}-A^{(2) *}$ and $A^{(12)}-A^{(12) *}$ are given by sum over cuts of the tree diagrams. This gives, in the notation of [11]:

$$
\begin{aligned}
A^{(1)}-A^{(1) *} & =A_{1}^{(1)}+A_{2}^{(1)}+A_{\underline{12}}^{(1)}, \quad A^{(2)}-A^{(2) *}=A_{1}^{(2)}+A_{2}^{(2)}+A_{\underline{2} 1}^{(2)}, \\
A^{(12)}-A^{(12) *} & =A_{1}^{(12)}+A_{2}^{(12)}+A_{\underline{1} 2}^{(12)}+A_{\underline{2} 1}^{(12)} .
\end{aligned}
$$

Here $A_{j_{1} \cdots j_{m}}^{\left(i_{1} \cdots i_{n}\right)}$ for $1 \leq i_{k} \leq 2,1 \leq j_{k} \leq 2$ represents sum over all cuts of $A^{\left(i_{1} \cdots i_{n}\right)}$ satisfying the following properties:

1. The cut can be viewed as a cut of the original graph contributing to the amplitude $A$. 
2. The cut crosses the $j_{1}, \cdots j_{m}$ 'th propagators in the set $\{1,2\}$ and possibly other propagators outside the set $\{1,2\}$.

On the other hand $A_{i_{1} j}^{\left(i_{1} \cdots i_{n}\right)}$ describes sum over cuts of $A^{\left(i_{1} \cdots i_{n}\right)}$ which pass through the $j$-th and $i_{1}$-th propagators in the set $\{1,2\}$ and possibly other propagators outside the set $\{1,2\}$, but which are not regular cuts of the original amplitude $A$ since, viewed in the context of the original graph, the $i_{1}$-th propagator carries energy across the cut in the wrong direction. Explicit diagrammatic representation of all the terms on the right hand side of (3.22) has been given in figures 6-15. In particular figures 8, 11, 14 and 15 describe contributions to $A_{\underline{1} 2}^{(1)}, A_{\underline{2} 1}^{(2)}, A_{\underline{1} 2}^{(12)}$ and $A_{\underline{2} 1}^{(12)}$ respectively. As is clear from the right hand sides of these figures, these are perfectly good cuts of the tree amplitude $A^{\left(i_{1} \cdots i_{n}\right)}$, even though the left hand sides of these figures show that they are not valid cuts of $A$.

We shall now write down a few identities following from the simple observation that a propagator cut twice has the same expression as the propagator cut once, since the cut passing through an external line has no effect. Therefore we have

$$
A_{2}^{(1)}=A_{2}^{(12)}, \quad A_{1}^{(2)}=A_{1}^{(12)}, \quad A_{\underline{1} 2}^{(1)}=A_{\underline{12}}^{(12)}, \quad A_{\underline{2} 1}^{(2)}=A_{\underline{2} 1}^{(12)} .
$$

These identities can be verified by explicitly examining the equalities of figures 7 and 13, 9 and 12, 8 and 14, and figures 11 and 15. Using (3.15), (3.20), (3.21), (3.22) and (3.23) we now get

$$
\begin{aligned}
A-A^{*} & =A_{1}^{(1)}+A_{2}^{(1)}+A_{\underline{12}}^{(1)}+A_{1}^{(2)}+A_{2}^{(2)}+A_{\underline{2} 1}^{(2)}-A_{1}^{(12)}-A_{2}^{(12)}-A_{\underline{1} 2}^{(12)}-A_{\underline{2} 1}^{(12)} \\
& =A_{1}^{(1)}+A_{2}^{(2)} .
\end{aligned}
$$

The diagrammatic representation of the right hand side, given by the sum of the left hand sides of figures 6 and figure 10, is shown in figure 16. We now see that this is precisely given by the sum of all possible cuts of the reduced diagram shown in figure 2 . In particular possible contributions from anomalous thresholds, represented by figure 7 and figure 9, cancel with the contributions from figures 13 and 12 and do not appear in the final expression. These cancellations are special cases of the general results described in eqs. (5.26)-(5.33) of [11].

We end this section with a few remarks:

1. Our analysis automatically includes other reduced diagrams that are obtained by contracting one or more propagators in figure 2. We simply have to set to zero all terms where the corresponding propagator is replaced by a cut propagator. For example if we take the triangle diagram obtained by contracting the propagator 3 in figure 2, the final answer for $A-A^{*}$ will include sum over only the second and third diagrams in figure 16.

2. This does not cover all the cases however. An example is shown in figure 17 where at the pinch poles on the same side come from non-adjacent propagators. We can analyze this by repeating the analysis, with the role of the momenta $\ell$ and $\ell+p_{C}$ in (3.14) now played by $\ell$ and $\ell-p_{A}-p_{B}$. The rest of the analysis proceeds as before, 


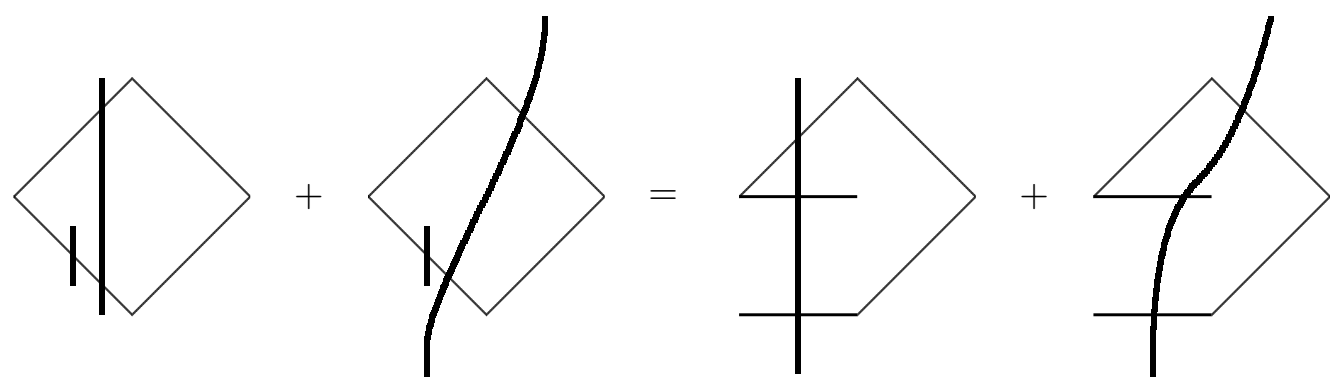

Figure 6. Contributions to $A_{1}^{(1)}$. The figure on the left hand side expresses it as a sum of cut diagrams of the original graph, with the propagator 1 replaced by the cut propagator. The right hand side shows this as a sum of cuts of a tree diagram in which the propagator 1 is replaced by a pair of incoming and outgoing lines. We have not shown the external states of the original amplitude $A$ in any of the diagrams.

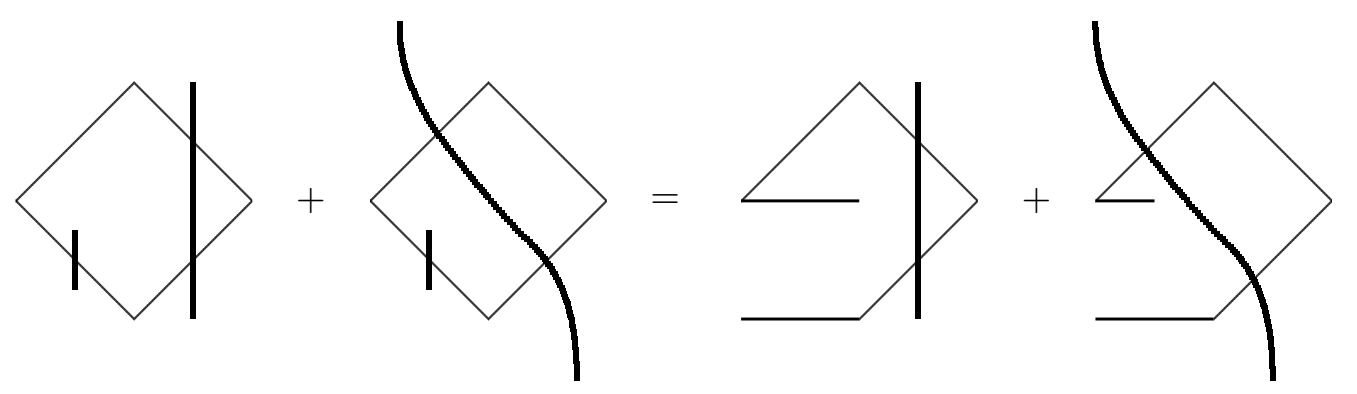

Figure 7. Contributions to $A_{2}^{(1)}$. Unless cancelled, this would represent contributions from anomalous threshold.

with the role of the set $\{1,2\}$ played by the set $\{1,4\}$. The final result for $A-A^{*}$, according to the general result of [11], will be given by $A_{1}^{(1)}+A_{4}^{(4)}+A_{14}^{(14)}$, which is simply the sum over all cuts of figure 17 . (The corresponding contribution $A_{12}^{(12)}$ was not present in the previous example since figure 2 has no cut that passes through both propagators 1 and 2.)

3. In our analysis we have assumed that for a given $\mathcal{R}$, the $\ell^{0}$ contour has a single pinch point to which two or more poles approach. We can also have more than one pinch on the $\ell^{0}$ contour, with two or more poles approaching each pinch point. Since after we factorize each propagator as in (3.9), each denominator factor is linear in $\ell^{0}$ and has a single zero, different denominator factors must be responsible for different pinches. Therefore we can divide the denominators into different sets, with the first set $S_{1}$ responsible for the first pinch, the second set $S_{2}$ responsible for the second pinch and so on. Different pinch points will have different reduced diagrams associated with them, since the list of singular propagators and the direction of energy flow through these propagators will depend on the pinch. 


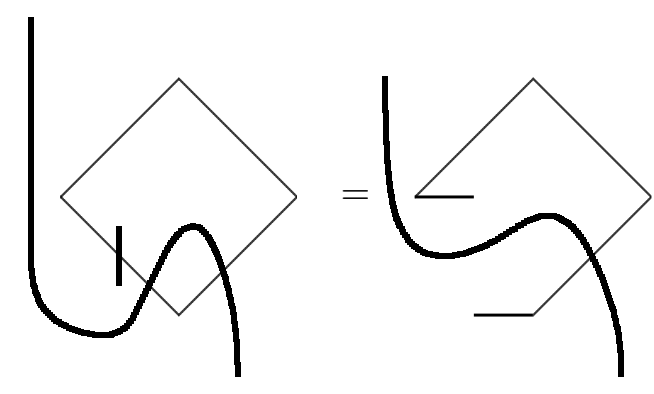

Figure 8. Contribution to $A_{\underline{1}}^{(1)}$. In the left hand side the cut seems to cross the propagator 1 of the original diagram in the reverse direction so that it appears as if the energy of the propagator 1 flows from the right of the cut to the left of the cut. However since the propagator 1 is already on-shell, the correct representation of the diagram is on the right hand side where it is represented as the cut of a tree diagram. In this representation there is nothing unusual.

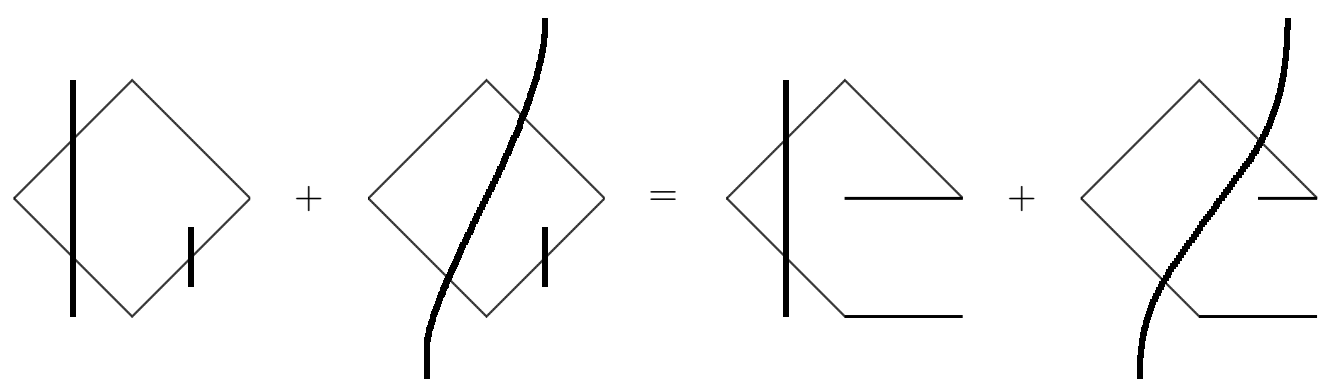

Figure 9. Contributions to $A_{1}^{(2)}$.
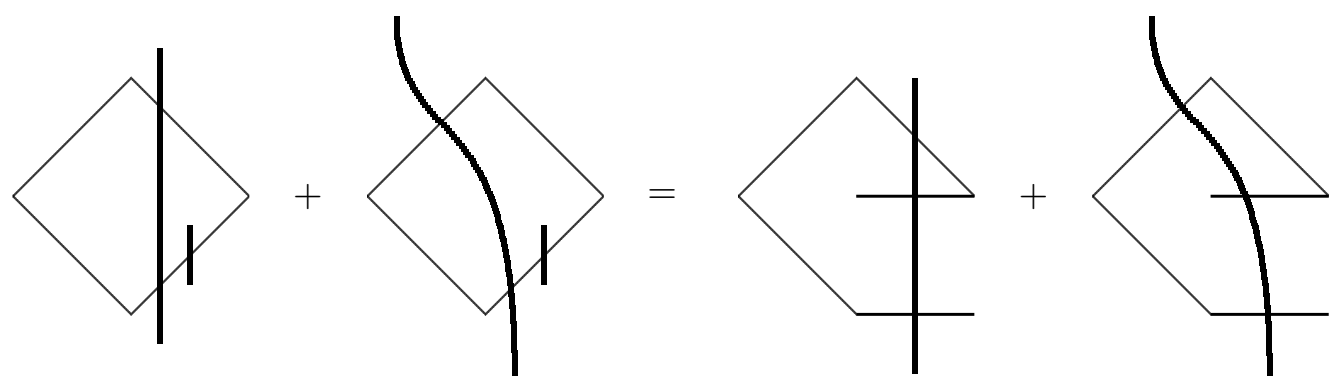

Figure 10. Contributions to $A_{2}^{(2)}$.

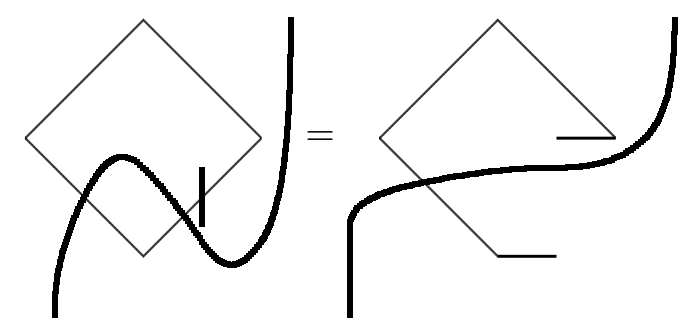

Figure 11. Contributions to $A_{\underline{2}}^{(2)}$. 


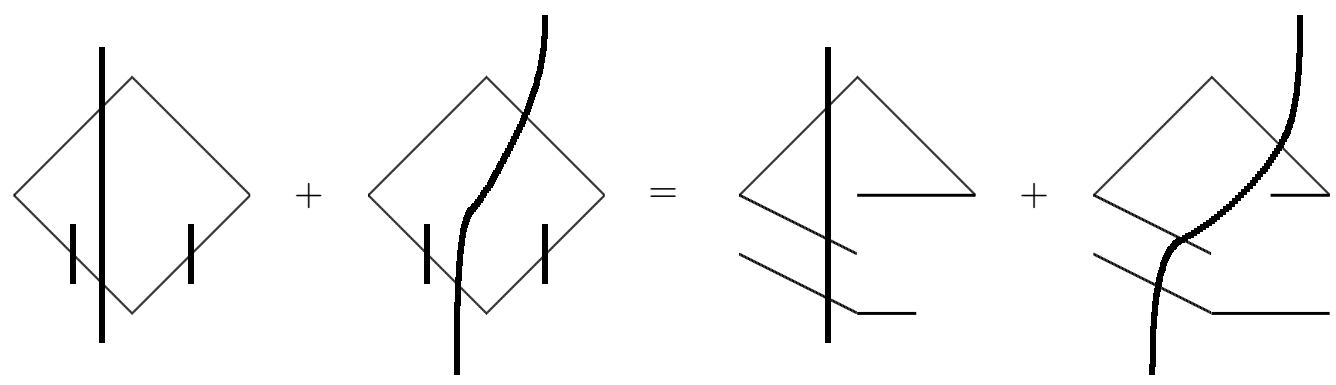

Figure 12. Contributions to $A_{1}^{(12)}$.
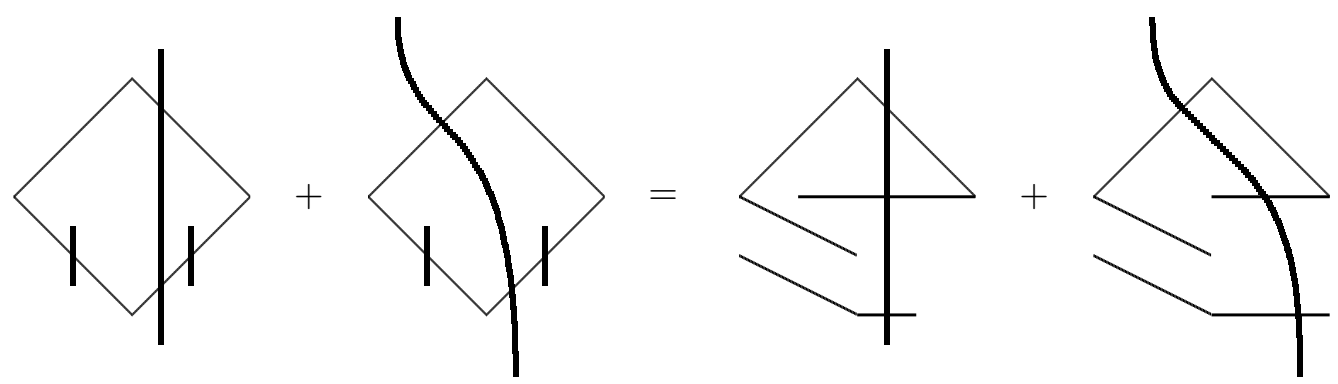

Figure 13. Contributions to $A_{2}^{(12)}$.

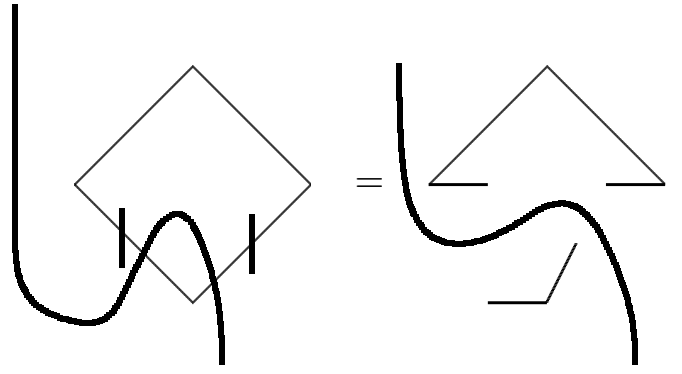

Figure 14. Contributions to $A_{\underline{12}}^{(12)}$.

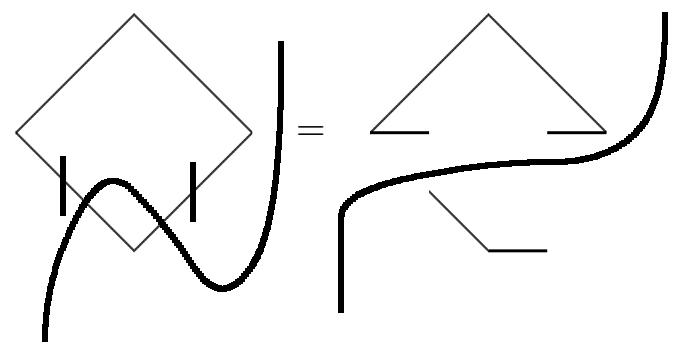

Figure 15. Contributions to $A_{\underline{2} 1}^{(12)}$. 


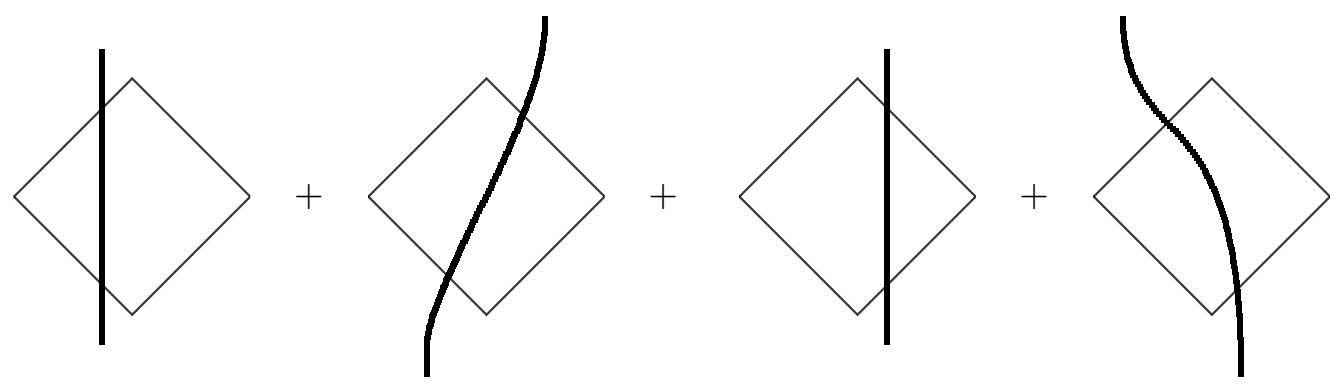

Figure 16. Complete contribution to $A-A^{*}$.

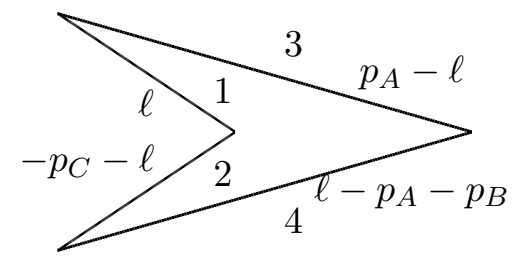

Figure 17. A reduced diagram in which non-adjacent propagators 1 and 4 have poles on the same side at the pinch. At the pinch the energy flows from left to right in each of the propagators. In the figure the momenta are labelled so that they flow from left to right.

We can now carry out the analysis by first treating the product of denominators in the set $S_{1}$ as in (3.14). The main difference will be that now the $\ell^{0}$ contour in $\widehat{A}$ is still pinched due to the other set of denominators belonging to $S_{2}, S_{3}, \cdots$ etc. The other terms contain delta functions that force $\ell^{0}$ to be at the first pinch and therefore the denominators in the other sets remain finite. These terms can be analyzed as before. We can now analyze $\widehat{A}$ by decomposing the second set of denominators, belonging to the set $S_{2}$, as in (3.9) and repeat the analysis. We continue this till we reach a stage where we have a sum of terms where in one term the contour is not pinched (the analog of $\widehat{A}$ ) and in the other terms the delta-function fixes $\ell^{0}$ at a pinch. The final result will then be given by the sum of cuts of all the reduced diagrams corresponding to all the pinches.

\section{Unitarity of tree diagrams}

Since the proof of cutting rules for the box diagram assumed the validity of cutting rules for connected and disconnected tree diagrams, we shall prove the cutting rules for tree diagrams in this section. The analysis is a straightforward application of sections 5.2.2 and 5.3 of [11].

We begin with the simple diagram shown in figure $18(\mathrm{a})$ with $p^{0}>0$. Its expression is given by

$$
A=\lambda^{2}\left(-p^{2}-m^{2}+i \epsilon\right)^{-1} .
$$




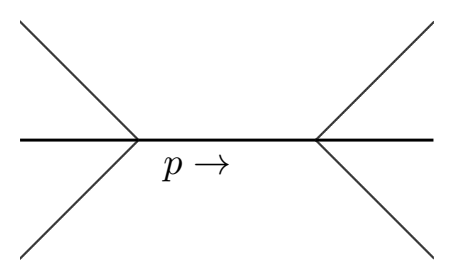

(a)

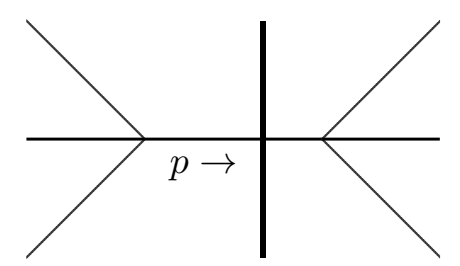

(b)

Figure 18. (a) A tree diagram in $\phi^{4}$ theory with six external lines and one internal propagator. In drawing this we have made an exception to our conventions and have drawn the external lines. The internal line has energy $p^{0}$ flowing from the left to the right. (b) Cut diagram of (a).

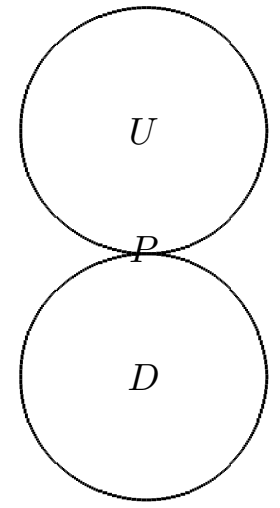

(a)

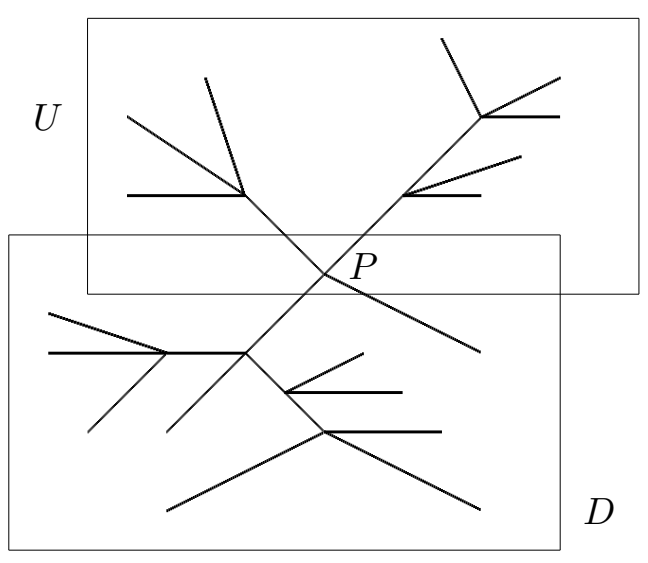

(b)

Figure 19. (a) Schematic representation of a generic connected tree diagram in which the blobs $U$ and $D$ themselves are connected tree diagrams. (b) Example of such a generic connected tree diagram.

Therefore

$$
A-A^{*}=\lambda^{2}\left(-p^{2}-m^{2}+i \epsilon\right)^{-1}-\lambda^{2}\left(-p^{2}-m^{2}-i \epsilon\right)^{-1}=\lambda^{2}(-2 \pi i) \delta\left(-p^{2}-m^{2}\right) .
$$

On the other hand the cut diagram shown in figure 18(b) has the same expression. (We can drop the $\theta\left(p^{0}\right)$ term from the cut propagator since $p^{0}$ has been chosen to be positive.) This proves the cutting rule for figure 18(a).

Next we shall prove the cutting rules for any connected tree amplitude assuming that it holds for all connected tree amplitudes with at least one less vertex. For this we follow closely the analysis of section 5.2.2 of [11] of 'one vertex reducible' diagrams. Let $P$ be any vertex of the amplitude to which at least two internal lines are connected. Then the general form of the diagram can be represented as in figure 19(a), with each of the blobs $U$ and $D$ describing some connected tree diagram. If $A_{U}$ and $A_{D}$ are the amplitudes associated 
with the tree diagrams $U$ and $D$ respectively, then the full amplitude $A$ is given by ${ }^{6}$

$$
A=\lambda^{-1} A_{U} A_{D}
$$

where the $\lambda^{-1}$ factor accounts for the fact that both $A_{U}$ and $A_{D}$ includes a factor of $\lambda$ from the vertex $P$ whereas in $A$ we have only one factor of $\lambda$ from the vertex. Therefore

$$
A-A^{*}=\lambda^{-1}\left\{A_{U} A_{D}-A_{U}^{*} A_{D}^{*}\right\} .
$$

Now since $A_{U}$ and $A_{D}$ are themselves connected tree amplitudes with less number of vertices than $A, A_{U}-A_{U}^{*}$ and $A_{D}-A_{D}^{*}$ are given by sum over cut diagrams of $U$ and $D$. We divide each of these cut diagrams into two classes: $\Delta_{U R}$ and $\Delta_{U L}$ will denote the sum over cut diagrams of $U$ for which the cut passes on the left and right of $P$ respectively, and similarly $\Delta_{D R}$ and $\Delta_{D L}$ will denote the sum over cut diagrams of $D$ for which the cut passes on the left and right of $P$ respectively. Therefore we have

$$
A_{U}-A_{U}^{*}=\Delta_{U L}+\Delta_{U R}, \quad A_{D}-A_{D}^{*}=\Delta_{D L}+\Delta_{D R}
$$

Using (4.5) and some trivial rearrangement of terms we can express (4.4) as

$$
A-A^{*}=\lambda^{-1}\left\{A_{U}^{*} \Delta_{D L}+\Delta_{U L} \Delta_{D L}+\Delta_{U L} A_{D}^{*}-\Delta_{U R} \Delta_{D R}+A_{U} \Delta_{D R}+\Delta_{U R} A_{D}\right\} .
$$

This can be verified e.g. by expressing both sides in terms of $A_{U}^{*}, A_{D}^{*}, \Delta_{U R}, \Delta_{U L}, \Delta_{D R}$ and $\Delta_{D L}$. The diagrammatic representations of the six terms in (4.6) have been shown in figure 20. Special attention should be paid to the minus sign of the fourth term on the right hand side of (4.6). This is compatible with the fourth term in figure 20 due to the $(-1)^{n_{R}-1}$ factor that multiplies each cut diagram, $n_{R}$ being the number of disconnected components on the right of the cut. If we denote by $n_{U R}$ and $n_{D R}$ the number of disconnected components on the right of the cut in $U$ and in $D$, then the product of the cut diagrams of $U$ and $D$ carries a factor of $(-1)^{n_{U R}-1+n_{D R}-1}$. On the other hand the fourth cut diagram of figure 20 carries a factor of $(-1)^{n_{U R}+n_{D R}-1}$. The two differ by a sign showing that the fourth diagram of figure 20 is indeed given by $-\lambda^{-1} \Delta_{U R} \Delta_{D R}$.

We now note that the six cut diagrams of figure 20 exhaust all possible cuts of the diagram 19(a). This shows that $A-A^{*}$ is indeed given by the sum over all cut diagrams of $A$ in accordance with the cutting rules.

Finally we turn to the proof of cutting rules for disconnected tree diagrams following the analysis of section 5.3 of [11]. Again the proof will proceed via induction, namely we shall prove the result assuming that it holds for diagrams with less number of vertices. For this let us suppose that the graph contains two pieces $B$ and $C$ that are disconnected from each other. This has been shown in figure 21. $B$ and $C$ themselves may be disconnected graphs, but each will contain less number of vertices and therefore satisfy cutting rules.

\footnotetext{
${ }^{6}$ Note that some of the external lines of $U$ at $P$ are internal lines of $D$ and some of the external lines of $D$ at $P$ are internal lines of $U$. Therefore they are generically off-shell. This does not have any effect on our analysis since the validity of cutting rules does not require the external lines to be on-shell.
} 

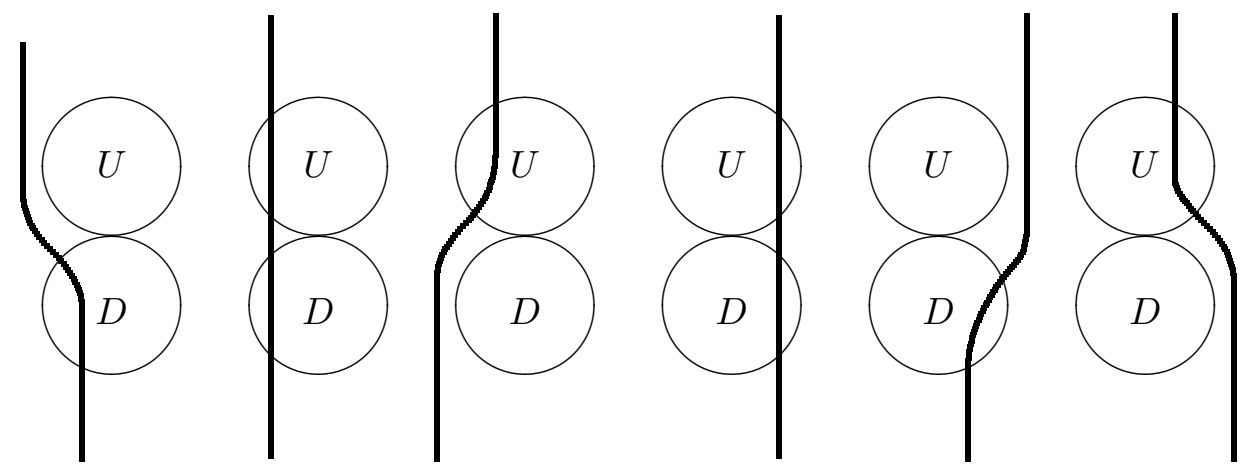

Figure 20. Diagrammatic representation of the six terms in (4.6). These can also be interpreted as cuts of figure $19(\mathrm{a})$.

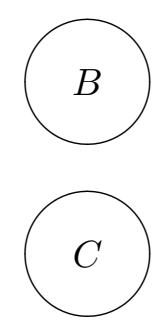

Figure 21. Schematic representation of a tree amplitude with at least two disconnected components. The blobs $B$ and $C$ represent tree amplitudes which themselves may have additional disconnected components.

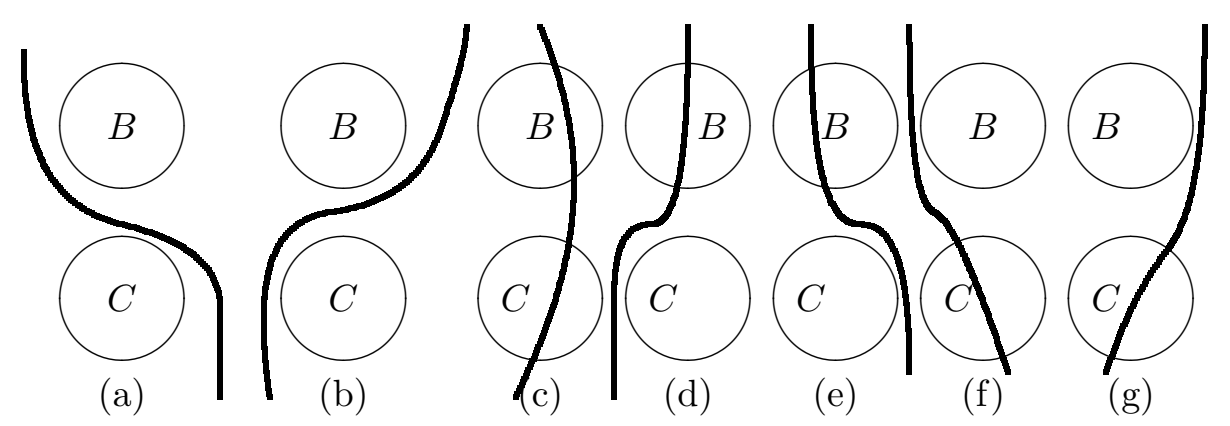

Figure 22. Diagrammatic representation of the seven terms in (4.8). These can also be interpreted as cuts of figure 21 .

Denoting by $B$ and $C$ the expressions for the amplitudes associated with the graphs $B$ and $C$, we have the full amplitude $A$ given by

$$
A=-i B C
$$

where the factor of $-i$ arises due to the fact that the total number of disconnected components of $A$ is equal to the sum of the number of disconnected components of $B$ and of $C$, and 
therefore due to the $(i)^{1-n_{c}}$ factor in the expression for the amplitude with $n_{c}$ disconnected components, the product $B C$ has one more factor of $i$ compared to $A$. This gives

$$
\begin{aligned}
A-A^{*}= & -i\left(B C+B^{*} C^{*}\right) \\
= & -i B^{*} C-i B C^{*}+i\left(B-B^{*}\right)\left(C-C^{*}\right)+i\left(B-B^{*}\right) C^{*}-i\left(B-B^{*}\right) C \\
& +i B^{*}\left(C-C^{*}\right)-i B\left(C-C^{*}\right) .
\end{aligned}
$$

The second step is the result of trivial algebraic manipulation. The seven terms in (4.8) can be diagrammatically represented as the seven cut diagrams shown in figure 22 . The additional minus signs in the contributions from figure 22(c), (d) and (f), given respectively by the third, fourth and sixth terms in (4.8), account for the fact that if $n_{R}$ denotes the number of disconnected components to the right of the diagram, then the sum of $n_{R}-1$ for the component diagrams differ from $\left(n_{R}-1\right)$ of the full diagram by 1 .

We now note that the seven terms in figure 22 are in one to one correspondence with the cuts of $A$. This proves the validity of the cutting rule for the disconnected amplitude $A$.

\section{Acknowledgments}

We would like to thank Alok Laddha and Terry Tomboulis for useful discussions. The research of R.P. was supported in part by Perimeter Institute for Theoretical Physics. Research at Perimeter Institute is supported by the Government of Canada through Industry Canada and by the Province of Ontario through the Ministry of Research and Innovation. This research of A.S. was supported in part by the J. C. Bose fellowship of the Department of Science and Technology, India.

Open Access. This article is distributed under the terms of the Creative Commons Attribution License (CC-BY 4.0), which permits any use, distribution and reproduction in any medium, provided the original author(s) and source are credited.

\section{References}

[1] R.E. Cutkosky, Singularities and discontinuities of Feynman amplitudes, J. Math. Phys. 1 (1960) 429 [INSPIRE].

[2] M. Fowler, Introduction to Momentum Space Integration Techniques in Perturbation Theory, J. Math. Phys. 3 (1962) 936.

[3] S. Mandelstam, Unitarity Condition Below Physical Thresholds in the Normal and Anomalous Cases, Phys. Rev. Lett. 4 (1960) 84 [InSPIRE].

[4] R.E. Cutkosky, Anomalous Thresholds, Rev. Mod. Phys. 33 (1961) 448.

[5] R. Zwicky, A brief Introduction to Dispersion Relations and Analyticity, in Proceedings of Quantum Field Theory at the Limits: from Strong Fields to Heavy Quarks (HQ 2016), Dubna Russia (2016), pg. 93 [arXiv: 1610.06090] [INSPIRE].

[6] P. Chin and E.T. Tomboulis, Nonlocal vertices and analyticity: Landau equations and general Cutkosky rule, JHEP 06 (2018) 014 [arXiv: 1803.08899] [INSPIRE]. 
[7] M.J.G. Veltman, Unitarity and causality in a renormalizable field theory with unstable particles, Physica 29 (1963) 186 [INSPIRE].

[8] G. 't Hooft and M.J.G. Veltman, Diagrammar, NATO Sci. Ser. B 4 (1974) 177.

[9] G.F. Sterman, An Introduction to quantum field theory, Cambridge University Press, Cambridge U.K. (1993).

[10] E.T. Tomboulis, Causality and Unitarity via the Tree-Loop Duality Relation, JHEP 05 (2017) 148 [arXiv: 1701.07052] [InSPIRE].

[11] R. Pius and A. Sen, Cutkosky rules for superstring field theory, JHEP 10 (2016) 024 [Erratum ibid. 1809 (2018) 122] [arXiv:1604.01783] [INSPIRE].

[12] F. Briscese and L. Modesto, Cutkosky rules and perturbative unitarity in Euclidean nonlocal quantum field theories, arXiv:1803.08827 [INSPIRE].

[13] D. Anselmi, Algebraic cutting equations, Annals Phys. 394 (2018) 294 [arXiv:1612.07148] [INSPIRE]. 